\title{
A Novel Planar Waveguide Laser
}

\author{
Dele Shi, Hongyan Xu, Xiujun Huang, Kaiming Wang, Bingchuan Du, Hongwen Zhou \\ Shandong Institute of Space Electronic Technology, Yantai, China \\ Email:sh_dl@163.com
}

How to cite this paper: Shi, D.L., Xu, H.Y., Huang, X.J., Wang, K.M.., Du, B.C. and Zhou, H.W. (2021) A Novel Planar Waveguide Laser. Optics and Photonics Journal, $11,326-331$.

https://doi.org/10.4236/opj.2021.118022

Received: June 29, 2021

Accepted: July 31, 2021

Published: August 3, 2021

\begin{abstract}
A new planar waveguide laser was demonstrated. The output energy of 400 $\mathrm{mW}$ was achieved by a single waveguide laser; the slope efficiency was $61 \%$. The single waveguide laser can expand to waveguide laser group and waveguide laser array to produce higher energy.
\end{abstract}

Keywords

Lasers, Solid-State, Laser Amplifiers

\section{Introduction}

Planar waveguide laser is a new potential technology combined with the advantages of fiber laser and Zigzag slab laser [1] [2], such as high extraction efficiency, good beam quality in waveguide direction, high stored energy, no nonlinear effect et al.. In this paper, a novel planar waveguide construct is put forward which adopts coupling mode of the pump light with internal total reflection. Also it can expand to waveguide laser group and waveguide laser array, which will obtain higher energy [3] [4] [5].

\section{Waveguide Laser Character}

The waveguide can be divided into 1-dimensional and 2-dimensional waveguide according to the optical limit dimension. The 1-dimensional waveguide is also called planar waveguide, usually composed of three mediums with different refractive index as cover layer $\left(\mathrm{n}_{0}\right)$, waveguide layer $\left(\mathrm{n}_{1}\right)$ and underlay layer $\left(\mathrm{n}_{2}\right)$ (Figure 1), which satisfy $n_{1}>n_{0}$ and $n_{2}$. The thickness of cover layer and underlay layer is far great than waveguide layer's. So the thickness is regard as infinity and beam can propagate in $\mathrm{z}$ and $\mathrm{y}$ direction, at the same time only $\mathrm{x}$ direction is limited.

The planar waveguide structure is usually produced by micro machining technology [6]. In the planar waveguide laser, waveguide structure is regarded as 


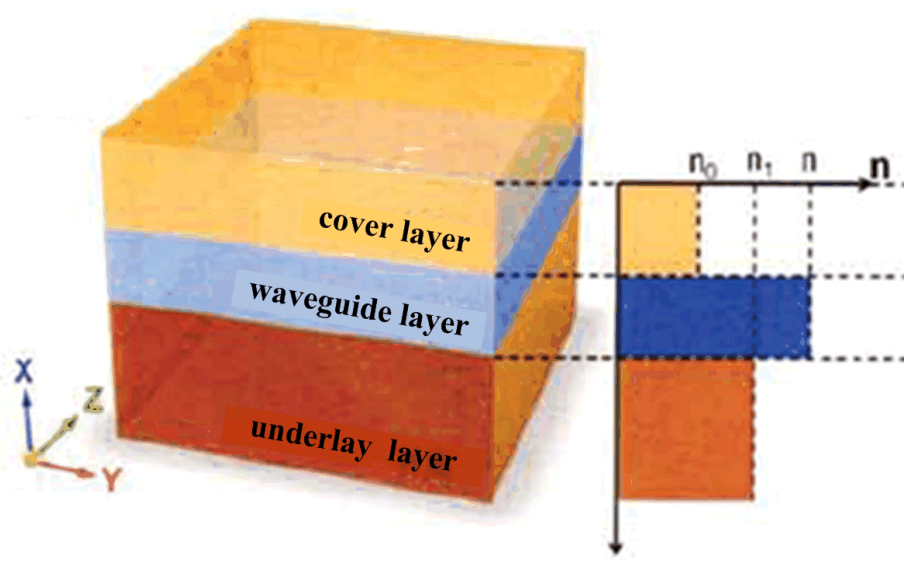

Figure 1. 1-Dimension waveguide.

resonator, and laser oscillation occurs by coupling the pumping beam with the waveguide resonator. The advantages of waveguide laser are shown as below.

1) The particle number reversal can be achieved easily in waveguide laser, because of the special pumping mode.

2) The thickness ratio of waveguide structure in horizontal and vertical direction, which makes it easier to dissipate heat.

3) Waveguide laser has little volume, pump threshold is less than bulk material. The ratio of pump threshold between them is shown as following equation.

$$
\frac{P_{\text {th_bulk }}}{P_{\text {th_wg }}}=\frac{V_{\text {eff_bulk }}}{V_{\text {eff_wg }}}\left[1-\frac{2 \alpha_{p} l(\kappa+1)}{(\kappa+1) L_{i}+2 N_{0} \sigma l}\right]^{-1}
$$

where: $V_{\text {eff } j}$ (j: bulk is bulk material, $w g$ is Waveguide) is the volume of effective transfer mode, $\alpha_{p}$ is the loss of waveguide transfer, $l$ is the length of amplifier medium, $L_{i}$ is the inherence loss of resonator cavity, $2 N_{o} \sigma l$ is the bring loss of re-absorb, $N_{o}$ is energy density of ground state, $\sigma$ is emission section of fluorescence, $\kappa$ is ratio of output coupler and back-forth loss.

\section{Waveguide Laser Designing}

In the waveguide laser, Nd:YAG ceramic is selected as solid laser medium, which easily be made into slice. Its structure was shown as Figure 2, laser emits along the z-axis, and pump light is coupled into the waveguide medium along the $\mathrm{y}$-axis. The thickness of the Nd:YAG ceramic was varied from $2 \mathrm{~mm}$ to $3 \mathrm{~mm}$ along the $y$ direction. As a result, the parasitic oscillation was suppressed in the $\mathrm{x}$ direction for the two nonparallel surfaces.

The scheme of single waveguide laser is shown in Figure 2. A laser diode (LD) stack with a divergence angle of 30 degrees in the fast axis was used as the pump source. A coupler, which was identical with the waveguide, was used to couple the pump beam into the Nd:YAG medium. The couple efficiency can be improved by adjusting the distance between the coupler and waveguide. As Figure 3 shown, the unabsorbed pump would be reflected into waveguide by the right angle reflect prism at the top of the waveguide medium. 


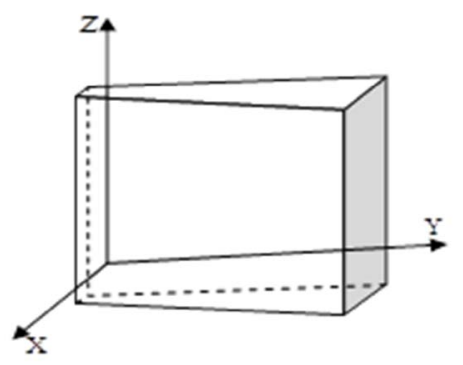

Figure 2. Schematic of a Nd:YAG trapeziform waveguide.

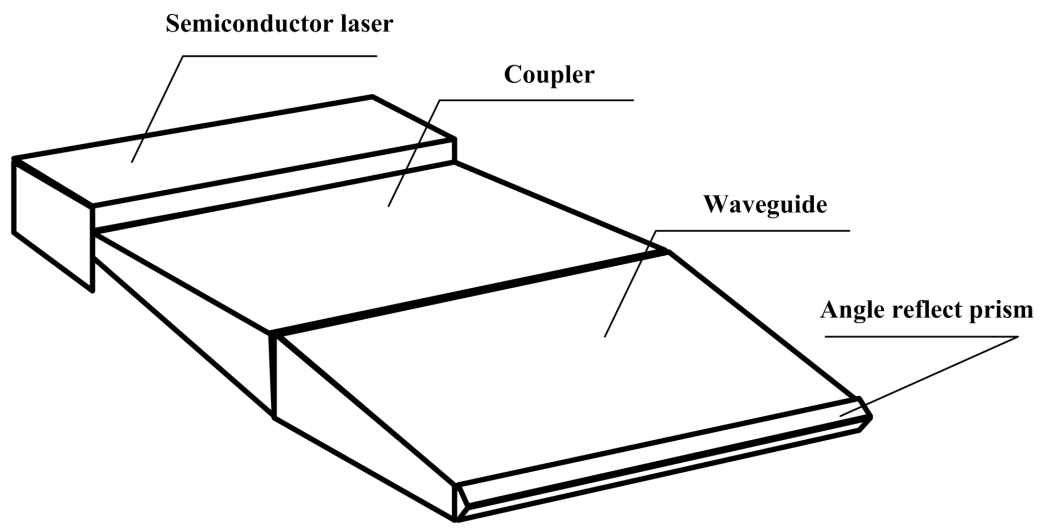

Figure 3. Single trapeziform waveguide laser.

The structure is also benefit for uniformity absorb of the pump. In general, when the pump light incidents into gain medium, its intensity decreases with the pump distance, which satisfies the equation as follows.

$$
I=I_{0} e^{-\alpha l}
$$

Expand the above formula in series and make a first-order approximate, we can obtain:

$$
I=I_{0}(1-\alpha l)
$$

It is shown that pump is uneven in the gain medium, which will lead to heat accumulation and heat damage, especially at the end face of the gain medium where the pump light is incident. However, pump light would decrease steadily and pump power density keep invariable in our waveguide laser, and follow as the equation:

$$
I=\frac{I_{0}(1-\alpha l)}{1-\beta l}
$$

where, $\alpha$ is absorb coefficient, $\beta$ is wedge angle of the gain medium. Absorb coefficient can be changed by two methods. One is changing doping concentration of the gain medium. The other is changing wavelength of the pump, such as 885 $\mathrm{nm}$, which is another absorb peak of Nd:YAG. Wedge angle can be changed by machining. When $\alpha$ and $\beta$ match consistently, $I$ is constant and pump is uniformity. The total internal reflection of the pump on the upper and lower sides of the waveguide can reduce the transmission loss of the pump and increase the 
absorption length.

Planar waveguide laser is showed as Figure 4. Figure 4(a) is single planar module. If multiple waveguide lasers are placed in parallel to form a series laser group, and the waveguide laser array can be formed by paralleling the series laser groups, as Figure 4(b) and Figure4(c) shown, which would achieve high power.

\section{Experiment and Discussion}

A schematic diagram of the waveguide laser setup is shown in Figure 5(a). A LD at $808 \mathrm{~nm}$ is used as a pump source. The pump laser is coupled to Nd:YAG waveguide slice after homogenization of coupler. The thickness of the Nd:YAG ceramic waveguide slice with doping concentrating of 1 at.\% decreases from $3 \mathrm{~mm}$ to $2 \mathrm{~mm}$, and the length and width are $21 \mathrm{~mm}$ and $10 \mathrm{~mm}$, respectively. Both the end faces of the slice in the laser output direction were flat and coated for antireflection at $1064 \mathrm{~nm}$ in order to reduce the intra-cavity loss. The mirror M1 was coated for high-reflection at $1064 \mathrm{~nm}$ and M2 was output coupling mirror with transmittance of $20 \%$ at $1064 \mathrm{~nm}$. TEC was employed to remove heat from the diode heat sink. The operation temperature was kept at about $25^{\circ} \mathrm{C}$.

The output of the waveguide laser was given in Figure 6. The output power increased almost linearly with the pumping power. The maximum output power of $400 \mathrm{~mW}$ was achieved with an incident pump power of $1.06 \mathrm{~W}$. The optical-to-optical conversion efficiency was as high as $37.7 \%$ and corresponding to slope efficiency of $61 \%$. No obvious evidence of saturation was observed from

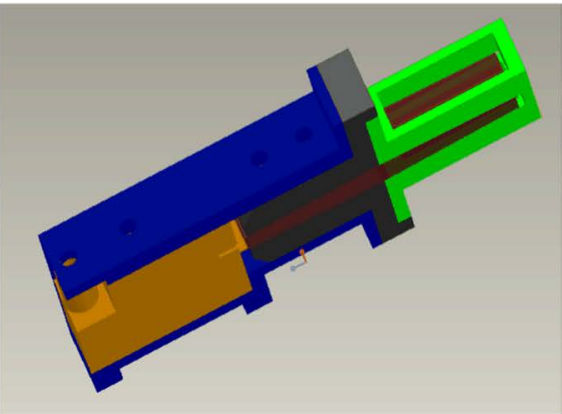

(a) single module

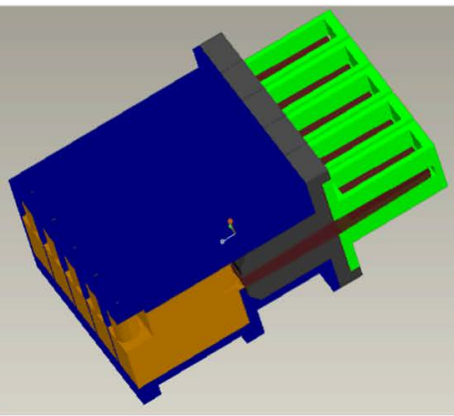

(b) waveguide laser group

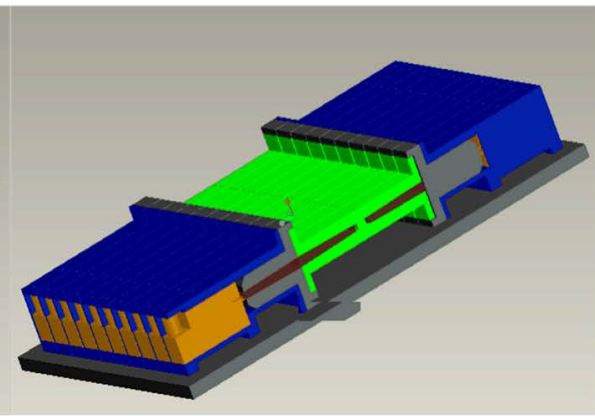

(c) waveguide laser array

Figure 4. Waveguide laser.

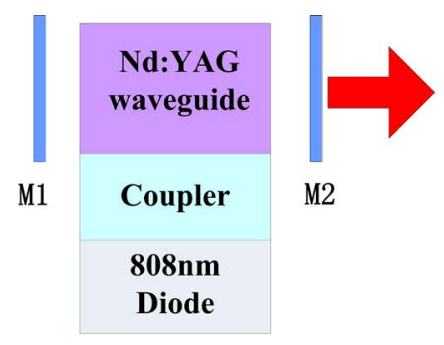

(a)

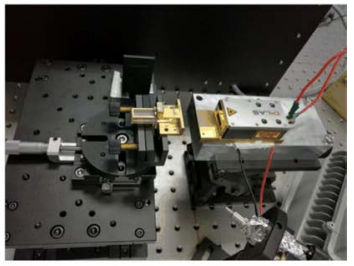

(b)

Figure 5. Experimental setup for waveguide laser. (a) Schematic diagram; (b) Waveguide laser setup. 


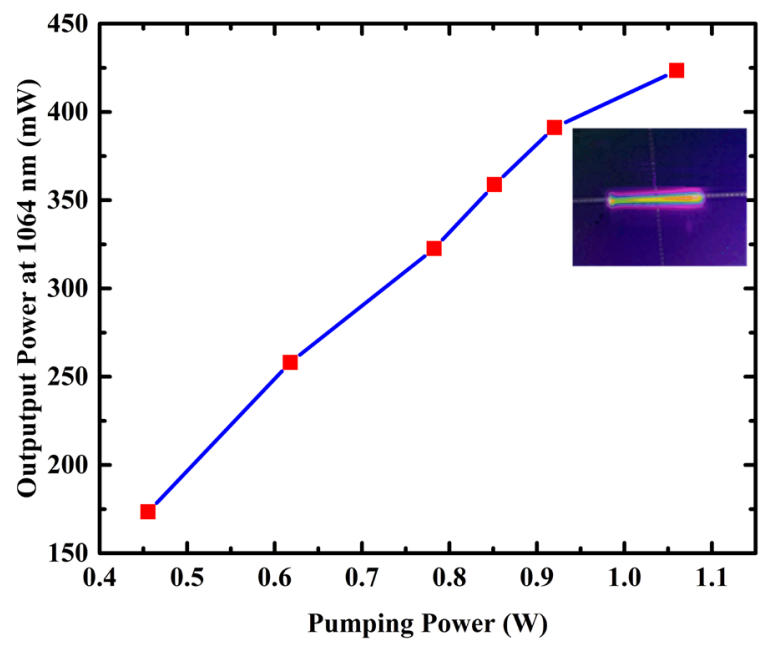

Figure 6. Output power versus pumping power.

the output curve, which means higher output power is possible if higher pump power is available. It also indicated that the new planar waveguide laser can achieve higher energy.

\section{Conclusion}

In summary, a novel planar waveguide laser was demonstrated. The performance of the waveguide laser was investigated, which indicates that the coupling mode of the pump light with internal total reflection can improve the coupling efficiency of pump light and achieve the purpose of uniform pumping, which can reduce pump threshold, reduce heat influence, improve the output laser beam quality and conversion efficiency. In addition, the single waveguide laser can be expanded to waveguide laser group and waveguide laser array, as shown in Figure 4, which will produce higher energy. It is important for the development of high energy laser.

\section{Conflicts of Interest}

The authors declare no conflicts of interest regarding the publication of this paper.

\section{References}

[1] Liao, C., Stegeman, G.I., Ceaton, C.T., Shoemaker, R.L., Valera, J.D. and Winful, H.G. (1985) Nonlinear Distributed Waveguide Coupler. Journal of the Optical Society of America A, 2, 590-594. https://doi.org/10.1364/JOSAA.2.000590

[2] Hettrick, S.J., Mackenzie, J.I., Harris, R.D., et al. (2000) Ion-Exchanged Tapered-Waveguide Laser in Neodymium-Doped BK7 Glass. Optics Letters, 25, 1433-1435. https://doi.org/10.1364/OL.25.001433

[3] Liao, C., Wang, J., Wei, Z. and Guo, J. (2007) Photon Emitting, Absorption and Reconstruction of Photons. Proceedings of SPIE-The International Society for Optical Engineering 27 th International Congress on High-Speed Photography and Photonics, Xi'an, 17-22 September 2006, 6279. https://doi.org/10.1117/12.725263 
[4] Eckhouse, V., Ishaaya, A.A., Shimshi, L., Davidson, N. and Friesem, A.A. (2006) Intracavity Coherent Addition of 16 Laser Distributions. Optics Letters, 31, 350-352. https://doi.org/10.1364/OL.31.000350

[5] Brunel, M., Floch, A.L., Bretenaker, F., Marty, J. and Molva, E. (1998) Coherent Addition of Adjacent Lasers by Forked Eigenstates Operation. Applied Optics, 37, 2402-2406. https://doi.org/10.1364/AO.37.002402

[6] Liao, C. and Stegeman, G.I. (1984) Nonlinear Prism Coupler. Applied Physics Letters, 44, 164-166. https://doi.org/10.1063/1.94722 